University of Nebraska - Lincoln

DigitalCommons@University of Nebraska - Lincoln

Faculty Publications from Nebraska Center for Research on Children, Youth, Families, and Schools
Children, Youth, Families \& Schools, Nebraska Center for Research on

2009

\title{
Intervention Implementation Integrity within Conjoint Behavioral Consultation: Strategies for Working with Families
}

\author{
Michelle S. Swanger-Gagne \\ University of Nebraska-Lincoln, MicSue17@aol.com \\ Andrew Garbacz \\ University of Oregon, andy.garbacz@wisc.edu \\ Susan M. Sheridan \\ University of Nebraska-Lincoln, ssheridan2@unl.edu
}

Follow this and additional works at: https://digitalcommons.unl.edu/cyfsfacpub

Part of the Child Psychology Commons, Counseling Psychology Commons, Developmental Psychology Commons, Family, Life Course, and Society Commons, and the Other Social and Behavioral Sciences Commons

Swanger-Gagne, Michelle S.; Garbacz, Andrew; and Sheridan, Susan M., "Intervention Implementation Integrity within Conjoint Behavioral Consultation: Strategies for Working with Families" (2009). Faculty Publications from Nebraska Center for Research on Children, Youth, Families, and Schools. 93. https://digitalcommons.unl.edu/cyfsfacpub/93

This Article is brought to you for free and open access by the Children, Youth, Families \& Schools, Nebraska Center for Research on at DigitalCommons@University of Nebraska - Lincoln. It has been accepted for inclusion in Faculty Publications from Nebraska Center for Research on Children, Youth, Families, and Schools by an authorized administrator of DigitalCommons@University of Nebraska - Lincoln. 


\title{
Intervention Implementation Integrity within Conjoint Behavioral Consultation: Strategies for Working with Families
}

\author{
Michelle S. Swanger-Gagné, S. Andrew Garbacz, and Susan M. Sheridan
}

Nebraska Center for Research on Children, Youth, Families and Schools, University of NebraskaLincoln, Lincoln, NE, USA

Corresponding author - Michelle S. Swanger-Gagné, email micsue17@aol.com

\begin{abstract}
Mental health services in school systems can take many forms. Behavioral consultation is one efficacious and commonly used form of indirect service delivery. Indirect service delivery models are unique in that an intermediate person, the consultee, provides treatment directly to a client. The effectiveness of the intervention depends in large part on the degree to which the consultee implements the intervention as designed. Families of children at-risk for school failure may experience challenges implementing an intervention developed through a consultation model. Some researchers have noted that the implementation of treatment plans is influenced by "events in the real world" including stress and limited economic and social resources (Cordray and Pion, in: R. R. Bootzin \& P. E. McKnight (eds.) Strengthening research methodology: Psychological measurement and evaluation, 2006); Watson et al. (in: Levensky and O'Donohue (eds.) Promoting treatment adherence: A practical handbook for health care providers, 2006). The purpose of this paper is to present descriptive findings from a large-scale consultation study that introduced unique strategies to promote intervention implementation integrity for children experiencing behavioral difficulties. Participants were separated into two groups, a general, mainstream group and a diverse, at-risk group (i.e., participants who were of low-income status, racially diverse, linguistically diverse, living in a single parent home, or who had less than a high school diploma). Consultants used general strategies (e.g., training and education) for maximizing intervention implementation integrity with all families and additional support strategies (e.g., regular contacts and additional home visits) with families at-risk. Reports via self-report and permanent product measures indicated families in both groups adhered
\end{abstract}


to intervention plans with high integrity when participating in $\mathrm{CBC}$. These strategies were illustrated in a case study format.

Keywords: treatment integrity, behavioral consultation, parent consultation, training consultants, risk factors

\section{Introduction}

Between $10 \%$ and $20 \%$ of children in American schools experience some form of mental health concern. Such concerns are manifest in social-emotional and behavioral problems that interfere with classroom instruction and student learning. Furthermore, they impact children's functioning in home and community settings, placing them at risk for school dropout, interaction with the criminal system, dependence on social services, and suicide. Only one-third of children receive needed services, leaving school personnel with the responsibility of addressing the vast majority of needs in order to minimize the impact on school functioning. Furthermore, few specialists are available to provide specialized services. School psychologists are among the most highly trained mental health practitioners in school settings; however, their availability is decreasing at the same time as needs escalate (Curtis, Grier, \& Hunley, 2004; Curtis, Hunley, \& Grier, 2004). The need for efficacious mental health services that provide access to evidence-based behavioral interventions to a number of students is paramount.

School-based behavioral consultation is an indirect form of service delivery wherein a specialist with expertise in social-behavioral interventions works collaboratively with a treatment agent (i.e., consultee, such as a teacher or parent) to address behaviors that interfere with learning. The consultation process is structured in a series of stages that allow the members of the consultation team (i.e., consultant, consultee[s]) to identify primary concerns experienced by a child, develop efficacious intervention plans intended to ameliorate the child's concerns, implement and support the delivery of treatment plan strategies in naturalistic (e.g., classroom, home) settings, monitor changes in the child's behaviors as a function of the intervention, and plan for maintenance to ensure ongoing benefit to the child. Conjoint behavioral consultation (CBC; Sheridan, Kratochwill, \& Bergan, 1996; Sheridan \& Kratochwill, 2008) is an extension of traditional consultation services. $\mathrm{CBC}$ focuses on joint, cooperative problem solving with teachers and parents sharing in the responsibility for implementing treatment plans across home and school, thereby promoting coordinated services and continuity across the settings within which children live. A great deal of empirical support is available documenting the efficacy of CBC at altering behavioral, social-emotional, and academic issues (Guli, 2005; Sheridan, Clarke, \& Burt, 2008; Sheridan, Eagle, Cowan, \& Mickelson, 2001).

$\mathrm{CBC}$ is, by definition, an indirect model of service delivery intended to enhance the skills of natural treatment agents (i.e., teachers, parents) who assume responsibility for implementing intervention plans. Therefore, an important consideration in CBC as a service model is the ability of individuals (often who are not trained in behavioral and mental health strategies) to deliver treatment plans accurately and effectively, in a manner that is both consistent with their design and has the potential to positively influence children's 
functioning. That is, the issue of intervention implementation integrity is central to the utility of CBC in addressing students' behavioral and mental health issues. Indeed, a treatment cannot achieve its desired effects if it not delivered, or if it is delivered in a manner that is inconsistent with its evidence base.

To ensure that students who are experiencing challenging mental health and behavioral issues have access to effective behavioral interventions, the fidelity with which teachers and parents implement treatment plans within the context of consultation services requires careful attention. The structure imposed through behavioral consultation allows for identifying and operationalizing target behaviors, conducting functional assessments, linking assessment data to intervention approaches, and evaluating the effects of interventions using single case research methods. However, little systematic attention is afforded to the specific strategies that consultants can use to ensure accurate and reliable implementation of treatment plan elements in natural settings (Sanetti \& Kratochwill, 2008). The effects of $\mathrm{CBC}$ and related behavioral interventions may be compromised in situations where certain environmental or contextual conditions complicate, or at best fail to support, appropriate implementation. Little is known currently about conditions that predict or are related to the ability of family members to implement $\mathrm{CBC}$-developed interventions aimed at supporting children's appropriate behaviors.

Research within the family therapy and parent training literatures indicates that certain circumstances may create difficulties for some families to follow through on treatment recommendations. Broad social and environmental factors influence parenting behavior and response to treatment. A significant correlate with treatment dropout and poor outcomes following parent training interventions is socioeconomic hardship (Henggeler, Melton, \& Smith, 1992; Kazdin, 1990; Kazdin, Mazurick, \& Bass, 1993; McMahon, Forehand, Griest, \& Wells, 1981). Families living in low socioeconomic conditions face numerous stressors (e.g., poverty, low education, lack of social support) that may interfere with their ability to implement parenting interventions, thereby compromising treatment effects. In addition, families living in low income conditions tend to experience higher levels of psychological distress (McLoyd, 1990). They may have diminished emotional reserves to deal with daily pressures and few support systems to combat added stressors. Indirect threats to children's emotional well-being are also present, resulting from less positive parenting strategies that are characteristic of families experiencing psychosocial stress (Garbarino, 1976; Stormshak et al., 2000). The challenges present in families with poor financial, emotional, and physical resources place children at risk for dire behavioral and learning outcomes.

The relationship between parenting practices and children's behavioral outcomes is unequivocal (Stormshak et al., 2000). Just as punitive, authoritarian practices predict behavioral problems in children, positive and constructive parenting is associated with child compliance and prosocial skills (Weiss \& Schwarz, 1996). Efforts to promote positive parenting skills require attention to stressors faced by families that may challenge implementation, or "events in the real world" including limited economic and social resources (Cordray \& Pion, 2006; Watson, Foster, \& Friman, 2006). However, strategies to support families in the implementation of evidence-based interventions, within a problem solving (i.e., consultation) framework, have not received adequate attention. Thus, currently little 
is known about consultation practices that promote intervention implementation for families requiring additional support (Sanetti \& Kratochwill, 2008).

\section{Purpose}

The purposes of this paper are twofold. First, we describe the degree to which families implement interventions as designed in a consultation study to promote positive behavioral outcomes for children experiencing behavioral difficulties. Multiple methods of assessing integrity are described and illustrated. Second, strategies used by consultants to maximize intervention implementation integrity of treatment plans by all families are discussed. Because families living in disadvantaged social circumstances required additional support for intervention implementation, methods used by consultants to support families with diverse challenges will be presented. One family will be highlighted in case study form to specify unique challenges present during consultation, with attention to methods used by the consultant to overcome those challenges. As such, this paper will inform practitioners of tactics for helping all consultees-including families living in disadvantaged social circumstances - to implement interventions as planned and to confront barriers to treatment implementation.

\section{Methods}

Data used in this investigation represent part of a larger, four-year study evaluating the efficacy of $\mathrm{CBC}$ as an intervention that addresses concerns of kindergarten through third grade students whose disruptive behaviors place them at risk for academic failure. To examine the rates of intervention implementation integrity at home for this investigation, only data for participants from the CBC condition were used.

\section{Participants, Recruitment, and Selection}

Participants were 62 parents and their children. All students were referred to the study by their teachers who indicated a need for further intervention due to behavioral concerns. Student participants were primarily male (75\%), and White, non-Hispanic (67\%). Students' ages ranged from 5 to 9 years, with a mean age of 6.72 years of age. Parents and/or legal guardians including immediate and extended family members were invited by school personnel to participate. The average age of parents in this sample was 35 , and more than half indicated receiving some college or post-secondary training (78\%). Participants were separated into two groups, a general mainstream group $(N=36)$ and a diverse group $(N=26)$. Participants who were of low-income status, racially diverse (i.e., non-white), linguistically diverse (i.e., English is not the primary language), living in a single parent home, or who had less than a high school diploma were considered to be demographically diverse relative to the mainstream group.

Teachers nominated students for participation in the larger efficacy study using a multiple-gate procedure and took place at the classroom level. Students were selected using the systematic screening for behavior disorders (SSBD) multiple-gate screening procedure (Walker \& Severson, 1990). The SSBD is a well-developed and researched screening approach used to identify students with or at risk for behavioral problems. A second method 
of qualification occurred via a researcher-developed, teacher-completed screening instrument that included three ratings of student behavior: severity of externalizing behaviors; frequency of externalizing behaviors; and degree of need for additional intervention (Glover, Sheridan, Garbacz, \& Witte, 2005). Thus, a child could participate in the project if he or she was identified as a child who exhibited behavior concerns by the SSBD or the researcher derived scale.

\section{Setting}

Training for CBC consultants occurred within a research center at a Midwestern university. Cases were conducted in 17 kindergarten, first, second, and third grade classrooms in the surrounding area, including public and parochial schools. The majority of CBC interviews were conducted in classrooms of participating teachers; some parent orientations and all home visits were conducted in homes. Interventions were implemented across home and school settings for students who were the focus of consultation. Given that the purpose of this study was to examine the extent to which families in CBC implement intervention plans as designed in collaboration with teachers and consultants, home-based intervention implementation will be reported here.

\section{Measurement of Treatment Integrity}

A multi-method approach was used to assess integrity, as outlined below. Each family received two integrity scores; an integrity score as evident by parent report of intervention implementation integrity and a consultant rating of intervention implementation integrity as represented on permanent products used by families during the intervention. ${ }^{1}$ Intervention implementation integrity was assessed and scored throughout the intervention phase of the consultation process.

\section{Parent Self-Report Form}

The parent self-report form was completed daily by parents and used to assess intervention implementation integrity at home. The parent self-report form was created after the plan development phase by the consultant to present the predetermined intervention as succinct steps. This form listed all the steps of the behavioral intervention plan clearly and objectively. Each day, parents self-recorded adherence to each step of the plan by indicating "yes" (indicating that the plan step had been completed), "no" (indicating that a plan step was not completed), or "not applicable" (NA; e.g., no occasion to deliver the step, child did not perform required behavior, change in schedule). Steps completed on the selfreport form were summed and an average of steps met was computed based on the total number performed divided by the total number possible, excluding NA responses. All parents in the treatment condition were asked to complete intervention self-report forms. If parents did not complete or return the self-report form, the data were not considered. Although this method accounts for instances in which parents indicate they did not complete a plan step, it did not account for instances in which parents failed to return the intervention implementation report forms. 


\section{Permanent Product Report Form}

Permanent products were completed by parents in the CBC treatment group daily for the duration of the intervention (i.e., at least 4 weeks of intervention). Specifically, charts were collected from parents on which evidence (e.g., stickers, notes, marks, checks) were made, demonstrating they implemented steps of the intervention. The products ultimately served as evidence of intervention implementation. Interventions that used products were homeschool notes, progress monitoring forms, positive reinforcement charts, compliance matrices, activity checklists, self-monitoring forms, charts, token economies, and time-out logs. For example, a consultant used a home-school note written by the parent to confirm that the parent completed relevant steps of her home plan for a particular day.

Permanent product report forms were developed in a manner consistent with the selfreport form. Consultants began with the self-report form and eliminated intervention steps that were not visible on available permanent products, to create the permanent product report form. Trained research assistants and consultants reviewed permanent products and completed the permanent product report forms to reflect parents' delivery of plan components as reported on permanent products, just as the parents indicated adherence on the self-report forms. Specifically, coders (i.e., consultants and research assistants) recorded parents' completion of intervention plan components as "yes," "no," or "not applicable." A rating of "yes" indicated that the plan step had been completed, a rating of "no" indicated that a plan step was not completed, and a rating of "not applicable" indicated the step could not be completed. Steps completed on the report form were summed and an average of steps met was computed based on the total number performed divided by the total number possible, excluding not applicable responses and intervention steps not visible on permanent products. A consultant and research assistant completed the permanent product record form and interrater reliability computed. Because these data are part of a larger study that includes participants across treatment (CBC) and control conditions, interrater reliability was calculated on a larger $(n=101)$ sample. For the full sample, the intraclass correlation with two raters for home intervention permanent products was .84 and percent exact agreement across raters was $89 \%$ exact agreement (Sheridan, Welch, Kwon, Swanger-Gagné, \& Garbacz, 2009).

\section{Consultation Procedures and Consultant Training}

Advanced graduate students in school and counseling psychology were trained to mastery in the principles and procedures of $\mathrm{CBC}$ using competency-based training methods. Prior to beginning casework, consultants' demonstrated mastery of $\mathrm{CBC}$ procedures by achieving an average of $90 \%$ of $\mathrm{CBC}$ interview objectives across two or more role plays. Competency-based training procedures, structured interviews, and consultation casework were based on Sheridan and Kratochwill (2008). Specifically, within each classroom, a consultant (i.e., trained graduate student) met with the teacher and two to three parents for approximately four to five conjoint consultation sessions over approximately 8 weeks via three phases: First, the Needs Identification and Needs Analysis (Building on Strengths) phase involved collecting background information from various sources; discussing objectives; reviewing student, family, and school strengths; prioritizing one to two target behaviors 
per student; identifying and defining needs, settings, and goals; conducting functional behavior assessments; discussing baseline data collection; and selecting general strategies for change. Next, the Intervention Development and Implementation phase involved developing a plan to address student needs; training parents and teachers to implement the behavior plan; implementing the behavior plan, providing consultant support to maximize integrity in the home and school settings and gathering information about the child's behavior and intervention implementation integrity. Finally, the Intervention Evaluation (Checking and Reconnecting) phase involved discussing objectives; discussing progress made toward goals; evaluating the plan(s); and determining needs for plan continuation, generalization, fading, and/or changes.

Multiple consultant trainings focused on enhancing implementation integrity of homebased behavioral interventions. Consultants attended an 8-week training program whereby they were trained using lecture and role-play methods. Training continued throughout consultation in weekly $1 \mathrm{~h}$ group supervision meetings which included the project director, a licensed psychologist, consultants with 1-4 years of consultation experience, and intermittently the primary project investigator and research project director. During group supervision consultants (a) problem-solved difficult situations (e.g., lack of time, lack of resources, differences in values, and beliefs about family roles) that challenged intervention implementation integrity and (b) generated strategies for maximizing home intervention implementation integrity through literature searches and clinical opinions.

Trainings concentrated on maximizing implementation integrity of home-based behavioral interventions through the use of intentional strategies (i.e., general and additional support strategies). A list of general strategies to promote high integrity in the home setting was developed from two sources: (a) a literature review that informed consultant training, and (b) narrative information reported by trained consultants. Furthermore, additional support strategies for promoting high intervention implementation integrity with families who experience socioeconomic hardships and other challenging conditions (i.e., distinct procedures that follow a culturally sensitive, family-centered model of practice; and are individualized to the families' beliefs, needs, and values) were identified using a similar method. These general and culturally sensitive strategies are presented in table 1.

Table 1. Strategies for maximizing home intervention implementation integrity

\begin{tabular}{ll}
\hline General strategies & Examples \\
\hline $\begin{array}{l}\text { Follow partnership model during intervention de- } \\
\text { velopment phase (Sheridan \& Kratochwill, 2008) }\end{array}$ & - Encourage family to share \\
& - Focus intervention on behaviors important to the \\
& - Integrate family's ideas in intervention plan \\
- & Ask family questions, encourage families to ask \\
& questions \\
- & $\begin{array}{l}\text { Discuss possible challenges to intervention im- } \\
\text { plementation and problem-solve } \\
\text { - Make intervention feasible yet still evidence- } \\
\text { based }\end{array}$
\end{tabular}


Provide rationale for collecting integrity data

Script and package intervention implementation integrity forms (Watson, 2004).

Train and educate family on intervention delivery with various techniques
- Explain impact of intervention implementation integrity on children academically, behaviorally, and socially

- Explain the purpose of forms (serves as reminder to consultee, self-monitoring tool of parent behavior, communication tool between consultant and consultee, measure, and evidence of intervention effect)

- Set expectations with consultees by informing them of how, when, and where integrity forms will be collected

- Make sure the intervention is of high quality

o Develop protocol of intervention steps

o Only state critical steps of intervention

o Standardize steps of each evidence-based intervention

o Permanent products must contain specific information for coding purposes (i.e., location on form for recording date used, parent initials, reward provided, privilege lost)

- Make sure the intervention and integrity forms are easy to use

o Make steps easy to read

o Use simple steps and simple recording method (e.g., yes, no, n/a format)

o Create reproducible forms

- Use identical integrity form for integrity monitoring via self-report, permanent product coding, and consultant observations and feedback

o Review integrity data collected via observations and phone/email/in-person contacts

o Using data presented on forms, give specific feedback on the consultant's performance (e.g., percentage of steps completed, incomplete steps, completed steps), and problem-solve problems that occurred during implementation

- Provide parents with training about implementation

o Didactic instruction

o Role-play

- Provide in vivo training (Sterling-Turner, Watson, Wildom, Watkins, \& Little, 2001; Taylor \& Miller, 1997)

o Model intervention implementation

o Practice intervention implementation

o Observe intervention implementation

o Coach in intervention strategy use 
o Provide performance feedback (Witt, Noell, LaFleur, \& Mortenson, 1997)

- Review the data on data collection forms with consultees regularly

- Give specific feedback (e.g., percentage of steps implemented, steps missed, strategies for increasing integrity)

- Graph integrity data and review data (Noell, Gresham, \& Gansle, 2002).

Strategies for maximizing implementation integrity with families from diverse backgrounds

Consider culturally sensitive procedures (Sheridan \& Kratochwill, 2008)

Focus on strategies that build trust between families and educators

Enhance communication between consultant and families

Implement family-centered approach throughout consultation process
- Ask questions about cultural values, family system, roles of family members

- Respect values when developing intervention

- Focus on family-identified needs when identifying needs and integrate family intervention ideas in intervention plan

- Intervene in setting important to family (e.g., church)

- Use inexpensive intervention

- Address diversity issues directly; discuss cultural differences and how theyimpact treatment plan

- Use cultural liaison if needed

- Get to know family

- Demonstrate respect for different cultural styles

- Gain buy-in and acceptability of consultation and intervention

- Communicate child's success at home to school consultee

- Use clear communication strategies

- Use descriptive and concrete terms

- Refrain from jargon

- Prepare with parents before meeting

- Ask for parents to share

- Use summary statements and clarifying statements

- Refrain from potential stereotype statements

- Use trained interpreter when necessary

- Train interpreters about consultation and communication

- Learn about preferred method of communication

- Make sure all information is provided in preferred language

- Listen and respond to needs

- Use open-ended questions to elicit family's perspectives

- Refrain from making assumptions

- Meet family "where they are at" 
Gather information about home setting and family system

Facilitate regular contacts and provide collective support

Adjust integrity data collection to improve ease of collection
- Provide positive feedback on strategies already in place

- Set obtainable goals

- Integrate intervention into family routine

- Conduct home visits and observe family routines to help determine/integrate appropriate strategies

- Include other family members; include them in all interactions

- Gather information about daily routines, cultural differences, family system

- Make frequent contacts outside of consultation meetings (multiple home visits, phone contacts, school meetings)

o Review forms, discuss integrity

o Praise efforts to implement intervention

o Model, practice, coach, provide feedback

o Discuss ideas for ways to incorporate steps that were not completed

- Specify how family's efforts to implement intervention with integrity led to child's improvements

- Provide information about additional community resources

- Help coordinate services

- Conduct parent group consultation meetings; group can serve as parent support group

- Develop easy-to-use and easy-to-read forms

- Translate all data collection forms and/or use symbols or visuals to communicate

- Use permanent product as measure of integrity

- Conduct consultant observations and record observation on report form

\section{Behavioral Interventions}

Behavioral interventions were implemented for at least 4 weeks during the conjoint plan implementation phase. Behavioral interventions consisted of three standard components. First, a communication component involved a system of regular contact (e.g., home/school note, scheduled email, regular phone calls) between home and school, consistent with the philosophy of CBC. This system was established to relay information about the child's behavior (e.g., progress toward goal, rewards earned). Second, a motivation component was included to reinforce a child's increase in positive or preferred behavior or decrease in disruptive behavior. Rewards for desired behavior were delivered in a specified format (e.g., grab bag, spinner, chart move, behavior contract). Third, a functional component was included in the intervention. After the functional assessment was completed and a hypothesized function determined (i.e., attention, escape, avoidance, sensory stimulation, skill), an intervention linked to the function of the undesired behavior was implemented (e.g., if 
attention was the function of interruptions, the teacher may be taught differential attention procedures). Additionally, similar behavioral intervention procedures or steps were standardized across children and used in the development of the intervention implementation integrity measures. Each intervention included between 4 and 12 steps.

\section{Data Collection and Estimates of Implementation}

Implementation integrity data were collected from families during the Plan Implementation phase via two methods: self-report and permanent product. Families completed the Parent Self-Report Form, for at least 4 weeks of the Plan Implementation phase. Additionally permanent products, which included paper documents produced during intervention implementation, were collected weekly by consultants. Examples of permanent products are homeschool notes, reward lists, behavior charts, progress monitoring forms, and time-out logs. Permanent products were collected at the school, home, or through the mail for at least 4 weeks during the Plan Implementation phase.

High (greater than 75\%) levels of implementation integrity were present across all cases. Specifically, participants in the mainstream group report adhered to $77 \%$ of intervention plan steps on self-report forms $(S D=26 \%)$ and $79 \%$ of intervention steps as evident by permanent product information $(S D=24 \%)$. Families in the at-risk group reported adhering to intervention plans with high integrity, even more so than families in the mainstream sample. Families representing diverse backgrounds reported completing $81 \%$ of intervention steps via self-report $(S D=17 \%)$ and $91 \%$ of intervention plan steps via permanent product $(S D=18 \%)$.

\section{Case Study}

The following CBC case study was characterized with many challenges; language and cultural barriers, limited home-school communication, and little financial and social resources. The family also experienced a unique transition as they moved from Africa to the United States as refugees. Nyawela, a 6-year old girl in first grade, lived in a small Midwestern city with her biological father and mother (i.e., Mr. and Mrs. Dulani) and four siblings. Her family moved from Sudan approximately 7 years prior as refugees and is now living in poverty as they adjust to life in America. Nyawela was referred for conjoint behavioral consultation services by her teacher, Mrs. Paul, who was concerned with Nyawela's inability to complete academic work accurately and regulate her emotions and behavior at school.

\section{Preconsultation}

During the orientation meeting, Nyawela's family was introduced to the consultation framework and to another family participating in CBC with the consultant and Mrs. Paul. Orientation was conducted with two families in order to condense orientation meetings and allow for opportunities to facilitate parent support across families. Both families were originally from areas other than the continental United States (i.e., Sudan and Puerto Rico). The consultant initiated discussion between the families in attempt to build support for each family and understand their similar circumstances. By fostering a relationship between two 
families, families could see they were not the only ones experiencing the challenges of living in a new country, speaking a language other than English, acculturating to the American culture, learning about the American education system, and receiving feedback from the school about their children not meeting academic, social, and behavioral expectations. After the meeting, the consultant spoke with a cultural liaison with the public school district and gathered information via the Internet sources to increase understanding of the Sudanese culture and refugee experience.

A home visit was conducted in attempt to build relationships and trust between the family and consultant and to gain information about the family roles, structure, values, beliefs, and expectations for Nyawela. The consultant sat with Mrs. Dulani and discussed her values and expectations. Mrs. Dulani stated she wanted Nyawela to perform well academically at school, listen to her directions at home, go to bed on time, and read. During the visit, Mrs. Dulani explained her husband worked full time as a social service case worker and went to school full time. She and the consultant discussed her role as a mother and Ms. Dulani said she was expected to care for the children, cook, and keep the house clean while her husband's role is to discipline the children. These roles have changed since they moved to the United States because Mr. Dulani is rarely home and Mrs. Dulani is expected to enforce the house rules. Mrs. Dulani expressed a need for toys, clothes, and financial resources for the children. The consultant and Mrs. Dulani inquired about community resources at the school and gained information about a parent support group for refugee families and a community agency that provided financial resources.

\section{Needs Identification and Analysis Phases}

Mrs. Dulani and Nyawela's teacher, Mrs. Paul, met with the consultant and spoke about Nyawela's strengths and concerns. Both Mrs. Paul and Mrs. Dulani shared concerns related to Nyawela's academic performance and decided to focus the intervention in building Nyawela's reading skills and ability to stay on-task and ask for help during reading class and homework time. She worked independently, did not ask for help, and eventually failed to complete assignments. After completing a functional behavioral assessment, it was agreed upon that Nyawela did not have the academic skills to complete the work, needed academic help and the skill of asking for help, and was therefore avoiding work. With this information, the consultant, Mrs. Dulani, and Mrs. Paul developed a behavioral intervention together with the shared goal of increasing work completion to $100 \%$ with $50 \%$ accuracy.

\section{Intervention Development}

The school intervention included "chunking" the reading worksheets, planning breaks and time to receive help throughout worksheet time, training skills for how to ask for help and appropriately escape work (ask for break), and self-monitoring of help-asking skills. The home intervention included developing a structured homework routine, having all children sit together and complete homework or color together at table, and completing a home-school note to aid in home-school communication. An activity checklist was developed which presented visual cues (pictures of activities) along with English titles to prompt completion of the homework routine/schedule and breaks between activities. Visual cues 
were used because the family had difficulty reading English and with pictures the family was more likely to follow through with using the checklist. The Sudanese culture is typically collective in nature, and the Dulani family shared that principle. As such, Mrs. Dulani valued family goals over individual goals. Mrs. Dulani expressed a need for a family checklist to be used with all children and therefore a family routine and checklist were developed for intervention purposes. The consultant developed a family checklist in hopes this would increase Mrs. Dulani's buy-in to the intervention and thus maximize integrity. Additionally, the home intervention appeared to be against Mrs. Dulani's cultural values of living in the moment and valuing present time. Mrs. Dulani expressed it was difficult for her to follow a schedule and arrive at appointments on time, but wanted to improve these skills as they are a value and necessity for living in America and supporting her children's education. The consultant attempted to build these skills by providing multiple reminders of appointments and using tasks or activities as references (e.g., the meeting is directly after school). The consultant again provided additional support to the family as a whole to build the relationship with the family and build skills that would help Mrs. Dulani implement the intervention and future interventions with integrity.

\section{Intervention Implementation Phase: Strategies for Maximizing Integrity throughout In- tervention Implementation/Intervention}

During the needs analysis phase, home intervention integrity self-report forms were scripted and packaged to include standardized steps for routine (activity checklist), scheduling breaks, skills training, and home-school note interventions. Steps were phrased simply and consistently on all forms. Although the family spoke Nuor, their written language was Arabic. Thus, the consultant offered to have the forms translated into Arabic, but Mrs. Dulani stated she wanted her and her children to practice English language skills and thus Nyawela reviewed the steps of the plan repeatedly with her mother, siblings, and the consultant. This provided Nyawela an opportunity to be a leader of the family and teach them English skills. Permanent products were also created and reviewed with the family. The activity checklist was used by all children to remember their schedule every afternoon. Each child drew his or her own personal symbol in each activity's box symbolizing activity completion. A home-school note was also developed to facilitate communication around home and school goal attainment. Goal attainment was represented by "smiley" faces and not meeting a goal was represented by a "frowny" face. Pictures were used in place of words to increase the likelihood of completion. Mrs. Dulani and Nyawela enjoyed completing the permanent products and understood the usefulness of the products after the consultant provided the family with a rationale. The consultant aimed to develop a useful product in hopes the family would implement the intervention.

After the development of the home intervention integrity self-report forms and permanent products, one parent training meeting was completed with Mrs. Dulani, Mrs. Paul, and the consultant. During the meeting, the consultant trained Mrs. Dulani and Mrs. Paul on every intervention procedure, role-played intervention implementation, and discussed possible barriers to treatment intervention implementation. Following the third meeting, home visits were conducted with Mrs. Dulani and sometimes extended family members who were visiting. All family members were included in intervention implementation 
training as to maximize the likelihood the intervention would be implemented by at least one adult in the family.

The first home visit included the consultant modeling the intervention and the use of permanent products and the self-report form. The second home visit involved Mrs. Dulani implementing the intervention and recording adherence to intervention steps on the selfreport form and permanent products. The consultant observed Mrs. Dulani implementing the intervention, coached Mrs. Dulani during implementation, and provided performance feedback. Throughout the visits the consultant praised Mrs. Dulani's attempts and helped her to see the importance of completing the permanent products. The consultant explained the activity checklist would help the family remember their roles and responsibilities after school and remind Nyawela to complete her homework which will eventually improve Nyawela's academic skills. The home-school note was an important component as it showed Mrs. Dulani how Nyawela was performing at school and if Nyawela is aware of her mother's commitment to her education, it is more likely that Nyawela will work to meet her goals. By providing a rationale to the intervention components, the consultant aimed to make the intervention and forms meaningful to the family and thus improve integrity. During all home visits, the family and consultant problem-solved barriers and adjusted the intervention and intervention integrity forms to make them more feasible and useful to the family. Between home visits the consultant contacted the family weekly to discuss Nyawela's progress, the family's well-being, and attainment of community resources.

The intervention was discussed weekly and feedback and problem-solving occurred over the phone during these contacts. The consultant also attempted to communicate every other week with Mr. Dulani, to review the home intervention and his role, and discuss ways he could support his wife at home. Mr. Dulani, the family, and the consultant decided Mr. Dulani would read the home-school note, praise Nyawela for meeting the home and school goals, and praise all children for completing homework, listening to their mother, and going to bed on-time. The consultant also supported the classroom intervention at least weekly and spoke with Mrs. Dulani before or after school. During the home visit and throughout the consultation process, the consultant continuously measured Mrs. Dulani's ability to implement an intervention plan and need for modeling, coaching, and feedback. The consultant also encouraged Mrs. Paul to speak with Mrs. Dulani in person before or after school to discuss Nyawela's progress because Mrs. Dulani preferred face-to-face communication versus written communication. These efforts to maximize integrity resulted in the family adhering to $100 \%$ of intervention steps over 4 weeks as reported by parent self-report. Interestingly, the family implemented the intervention with less integrity (i.e., $46 \%$ adherence) as measured by the permanent product. It was very difficult for the family to adapt their typical lifestyle and complete a checklist or schedule on paper every day.

\section{Intervention Evaluation}

The final home visit was conducted to evaluate the intervention and the family's satisfaction and acceptance of consultation and the interventions. Improvements were seen in Nyawela's ability to focus and complete work with accuracy at school. On average, the teacher reported Nyawela completed work with $45 \%$ accuracy before the intervention and 
$80 \%$ accuracy after intervention implementation. She was meeting the goal of $100 \%$ completion and 50\% accuracy every day and therefore earning choice time. In addition, Nyawela's teacher reported that she asked for help more frequently and appeared more confident in her academic skills. The teacher also generalized the intervention techniques to math class. At home, Mrs. Dulani reported fewer problem behaviors at home as illustrated on the Parent Daily Report (PDR; Chamberlain \& Reid, 1987). Before the home intervention was implemented, Mrs. Dulani reported an average of four problem behaviors per day and during the behavioral plan she reported an average of one problem behavior per day. Mrs. Dulani expressed happiness with Nyawela's skill development and academic performance throughout the behavioral intervention. Mrs. Dulani improved her skill of establishing a homework routine at home, communicating with school, and praising her children for attaining goals. She also increased the number of school meetings she attended and began arriving on-time to meetings. Future ideas for maintaining academic success and home-school collaboration were discussed. Mrs. Dulani appeared to understand her role as a mother can be to teach her children at home and that in the United States of America it is appropriate for her to speak with school regularly about her children's progress. The consultant praised Mrs. Dulani's accomplishments and growth in hopes to empower her to continue taking an active role in her child's education at home and school.

\section{Discussion}

Children's mental health is dependent on experiences in various settings (e.g., home, school). School mental health professionals understand the effect of the home environment on a child's development and are knowledgeable about the best practice of home-school collaboration (Raffaele \& Knoff, 1999). When schools and families collaborate, they often develop interventions to be implemented at home and school and communication ends. Follow-up procedures about the integrity with which families implement interventions at home and methods to reduce barriers they experience during implementation rarely exist. If an intervention is not being implemented as designed, it may not have the intended effects (Mowbray, Holter, Teague, \& Bybee, 2003). Thus, it is important for educators to encourage families to self-monitor their adherence to intervention plans, continue communication with family members, and supportively coach families during intervention implementation.

The current mental health and educational literatures lack research measuring treatment integrity in the homes of children who exhibit disruptive behavior, one of the most influential environments where children learn (Kellaghan, Sloane, Alvarez, \& Bloom, 1993). This paper expanded the literature base by measuring the rate in which mainstream and at-risk families adhere to home behavioral interventions developed in a conjoint behavioral consultation model of service delivery. In addition, a description of general and specific strategies used by consultants to increase adherence to behavioral interventions and strategies employed to increase adherence are provided. 


\section{Observations from Descriptive Methods}

High levels of intervention implementation integrity were noted in the home setting across multiple sources (i.e., families via parent-report and coders of permanent product data) indicating that families seem to be responsive to tactics used by consultants to maximize treatment integrity. Families also appear to adhere to the steps of behavioral interventions regardless of the integrity measure used, with families reporting slightly higher integrity when recording integrity on a permanent product. Interestingly, the case study illustrated a unique example of a family who indicated more adherence to the intervention plan when reporting on the self-report form (i.e., $100 \%$ adherence) and less adherence when reporting on the permanent products (i.e., $46 \%$ adherence). This family may be a unique example as they did not find written permanent product components of the intervention (e.g., activity checklist) valuable. The consultant also provided guidance in completing the self-report forms. Specifically, the permanent products may be culturally loaded in that people from other cultures may not be accustomed to recording information or understand its importance. In sum, permanent products are a feasible and useful way of measuring treatment integrity because families naturally use the products as they implement interventions (Sanetti \& Kratochwill, 2008). Additionally, the high integrity levels reported on permanent products (with the exception of the case study) may suggest that parents are not overreporting on self-report measures. An exception may be when permanent products are used to represent implementation integrity for families of diverse cultures.

This paper presented intervention implementation data for families experiencing challenging life circumstances. In addition, a case study for a family of refugee status and who was living in poverty was presented to provide an example for how specific strategies to increase implementation could be used for a family at-risk. Limited research has investigated intervention implementation integrity for families experiencing struggles such as poverty, refugee status, and immigrant status. Intervention implementation integrity results indicated families at-risk are able to face environmental challenges and implement behavioral interventions as designed with the support of a consultant. When reviewing results of each integrity measure, the families at-risk reported implementing intervention with higher integrity than the mainstream group of families, indicating consultant efforts to promote integrity were especially helpful at promoting high integrity for these families. Consultant efforts to collaboratively develop meaningful interventions that were sensitive to the family's beliefs and values, provide additional support through frequent contacts, home visits, and coaching during intervention implementation, and adjust intervention integrity data collection as to improve ease of use appear to increase home intervention implementation integrity. It is possible that the additional strategies (e.g., more home visits and contacts) used by consultants can effectively improve intervention implementation for all families. The case study approach necessitates future research, employing a multimethod approach, replicate this finding with other families identified as "at-risk." School mental health professionals can use similar procedures to maximize integrity when collaborating with families to increase adherence to behavioral interventions implemented at home. 


\section{Limitations and Future Research Directions}

The collaborative model of service delivery and consultant training presented were implemented in a large funded investigation and future research must begin to understand the generalizability and feasibility of the model outside the context of research investigations. Several time-intensive strategies to increase treatment integrity were presented, such as home visits and frequent contacts. It is unknown if such strategies are realistic outside the context of a large, funded study. Further exploration is needed to understand and clarify the amount of hours consultants spend supporting intervention integrity across both general and additional support conditions. Resources may not be available for educators to implement all strategies noted in this article and the effect of each strategy on increasing intervention implementation adherence must be examined. Future research must explore further resources that are needed and specific procedures that are critical to intervention implementation. The cost-effectiveness of such intervention implementation integrity support practices needs to be assessed in future studies.

The current article used a descriptive and case study approach to data analysis. Future research should examine integrity and the variables that may influence levels of treatment integrity experimentally. Specifically, future research could attempt to measure the impact of disadvantaged social settings on the degree to which families follow a behavioral intervention protocol. Likewise, future research should focus on measuring and evaluating treatment integrity more broadly. Although this investigation employed a multisource approach to evaluate treatment integrity, the focus of the multiple sources was on adherence to behavioral intervention implementation. For example, dosage, quality of, and responsiveness to implementation are additional dimensions of integrity influencing service delivery (Dane \& Schneider, 1998).

Standardized measures of consultant strategies and intervention implementation integrity need to be developed and used in future research and psychometric properties of such measures must be evaluated. In the current study, strategies used by consultants to promote treatment integrity were collected from consultant narrative reports, in addition to a review of previous research investigating treatment integrity. Standardized measures of consultant strategies for maximizing integrity are needed. Measures of intervention implementation integrity used in the current article have been used previously in research, but evidence of their psychometric properties is limited (Sheridan et al., 2009). Furthermore, the treatment integrity estimates presented in the study did not include data from parents who did not return self-report forms and permanent products; integrity estimates may overestimate the actual implementation integrity score. As such, psychometric properties of future measures of integrity and strategies used by consultants to improve integrity should be examined.

This paper focused on treatment integrity at home. Similar issues with regard to measurement and evaluation should be explored at school. Furthermore, the link between home and school treatment integrity and its effects on student performance have not been systematically examined. This is an important step in understanding how the continuity of services across settings may affect student performance. 


\section{Conclusion}

The current paper is the beginning of a line of research needed to better understand treatment integrity, factors impacting integrity, and the most effective strategies for supporting families during treatment implementation, within a consultation context. The paper begins to explore the integrity in which families implement interventions at home when they have a consultant training and supporting them in a sensitive and family-centered manner. Results from this investigation suggest families can implement interventions with integrity when they are given the skills and support to do so. Furthermore, to effectively support families, it may be helpful for school mental health professionals to assume the role as family collaborator as a way to empower families to meet such expectations.

Acknowledgments - This work was developed with partial support from grants awarded to the third author (US Department of Education Grant Numbers H325D030050 and R305F05284).

\section{Note}

1. Interested readers can contact the corresponding author for samples of self-report forms, permanent product report forms, and permanent products.

\section{References}

Chamberlain, P., \& Reid, J. B. (1987). Parent observation and report of child symptoms. Behavioral Assessment, 9, 97-109.

Cordray, D. S., \& Pion, G. M. (2006). Treatment strength and integrity: Models and methods. In R. R. Bootzin \& P. E. McKnight (Eds.), Strengthening research methodology: Psychological measurement and evaluation (pp. 103-124). Washington, DC: American Psychological Association.

Curtis, M. J., Grier, J. E. C., \& Hunley, S. A. (2004a). The changing face of school psychology: Trends in data and projections for the future. School Psychology Review, 33, 49-66.

Curtis, M. J., Hunley, S. A., \& Grier, E. C. (2004b). The status of school psychology: Implications for a major personnel shortage. Psychology in the Schools, 41, 431-442.

Dane, A. V., \& Schneider, B. H. (1998). Program integrity in primary and early secondary prevention: Are implementation effects out of control? Clinical Psychology Review, 18, 23-45.

Garbarino, J. (1976). A preliminary study of some ecological correlates of child abuse: The impact of socioeconomic stress on mothers. Child Development, 47, 178-185.

Glover, T., Sheridan, S. M., Garbacz, S. A., \& Witte, A. (2005). Behavior severity, behavior frequency and need for intervention screening tool. Unpublished scale.

Guli, L. A. (2005). Evidence-based parent consultation with school-related outcomes. School Psychology Quarterly, 20, 455-472.

Henggeler, S. W., Melton, G. B., \& Smith, L. A. (1992). Family preservation using multisystemic therapy: An effective alternative to incarcerating serious juvenile offenders. Journal of Consulting and Clinical Psychology, 60, 953-961.

Kazdin, A. E. (1990). Premature termination from treatment among children referred for antisocial behavior. Journal of Child Psychology and Psychiatry, 31, 415-425. 
Kazdin, A. E., Mazurick, J. L., \& Bass, D. (1993). Risk for attrition in treatment of antisocial children and families. Journal of Clinical Child Psychology, 22, 2-16.

Kelleghan, T., Sloane, K., Alvarez, B., \& Bloom, B. S. (1993). The home environment and school learning: Promoting parental involvement in the education of children. San Francisco: Jossey-Bass.

McLoyd, V. C. (1990). The impact of economic hardship on Black families and children: Psychological distress, parenting and socioemotional development. Child Development, 61, 311-346.

McMahon, R. J., Forehand, R., Griest, D. L., \& Wells, K. C. (1981). Who drops out of treatment during parent behavioral training? Behavioral Counseling Quarterly, 1, 79-85.

Mowbray, C. T., Holter, M. C., Teague, G. B., \& Bybee, D. (2003). Fidelity criteria: Development, measurement, and validation. American Journal of Evaluation, 24, 315-340.

Noell, G. H., Gresham, F. M., \& Gansle, K. A. (2002). Does treatment integrity matter? A preliminary investigation of instructional implementation and mathematics performance. Journal of Behavioral Education, 11, 51-67.

Raffaele, L. M., \& Knoff, H. M. (1999). Beginning school ready to learn: Parental involvement and effective educational programs. School Psychology Review, 28, 448-466.

Sanetti, L. M. H., \& Kratochwill, T. R. (2008). Treatment integrity in behavioral consultation: Measurement, promotion, and outcomes. International Journal of Behavioral Consultation and Therapy, 4, 95-113.

Sheridan, S. M., Clarke, B. L., \& Burt, J. D. (2008). Conjoint behavioral consultation: What do we know and what do we need to know? In W. P. Erchul \& S. M. Sheridan (Eds.), Handbook of research in school consultation: Empirical foundations for the field (pp. 171-202). Mahwah, NJ: Lawrence Erlbaum.

Sheridan, S. M., Eagle, J. W., Cowan, R. J., \& Mickelson, W. (2001). The effects of conjoint behavioral consultation: Results of a four-year investigation. Journal of School Psychology, 39, 361-385.

Sheridan, S. M., \& Kratochwill, T. R. (2008). Conjoint behavioral consultation: Promoting family-school connections and interventions (2nd ed.). New York, NY: Springer Publishing.

Sheridan, S. M., Kratochwill, T. R., \& Bergan, J. R. (1996). Conjoint behavioral consultation: A procedural manual. New York: Plenum.

Sheridan, S. M., Welch, G., Kwon, K., Swanger-Gagné, M. S., \& Garbacz, S. A. (2009). Fidelity measurement in consultation: Psychometric issues and preliminary examination. School Psychology Review.

Sterling-Turner, H. E., Watson, T. S., Wildom, M., Watkins, C., \& Little, M. (2001). Investigating the empirical relationship between training type and treatment integrity. School Psychology Quarterly, 16, 56-67.

Stormshak, E. A., Bierman, K. L., McMahon, R. J., Lengua, L. J., \& the Conduct Problems Prevention Research Group. (2000). Parenting practices and child disruptive behavior problems in early elementary school. Journal of Clinical Child Psychology, 29, 17-29.

Taylor, J., \& Miller, M. (1997). When timeout works some of the time: The importance of treatment integrity and functional assessment. School Psychology Quarterly, 12, 4-22.

Walker, H. M., \& Severson, H. H. (1990). Systemic screening for behavior disorders (SSBD): Further validation, replication, and normative data. Remedial and Special Education, 11(2), 32-46.

Watson, T. S. (2004). Treatment integrity. In T. S. Watson \& C. H. Skinner (Eds.), Encyclopedia of school psychology (pp. 356-358). New York, NY: Springer Publishing. 
Watson, T. S., Foster, N., \& Friman, P. C. (2006). Treatment adherence in children and adolescents. In E. R. Levensky \& W. T. O'Donohue (Eds.), Promoting treatment adherence: A practical handbook for health care providers (pp. 1-14). Thousand Oaks, CA: Sage Publications, Inc.

Weiss, L. H., \& Schwarz, J. C. (1996). The relationship between parenting types and older adolescents' personality, academic achievement, adjustment, and substance use. Child Development, 67, 21012114.

Witt, J. C., Noell, G. H., LaFleur, L. H., \& Mortenson, B. P. (1997). Teacher use of interventions in general education settings: Measurement and analysis of the independent variable. Journal of Applied Behavior Analysis, 30, 693-696. 